CASE REPORT

\author{
V.V. Pai \\ L.H. Lowe \\ M. Castillo \\ B.Y. Huang \\ L. Shao
}

\section{Posterior Fossa Dermoid Cysts in Association with Klippel-Feil Syndrome: Report of Three Cases}

SUMMARY: Although the association of spinal lumbosacral dysraphism and congenital spinal dermoid tumors is well known, the association of craniocervical spinal anomalies and posterior fossa dermoids has only been recognized recently. Advances in imaging technology and awareness of the association likely contribute to an increase in recently reported cases.
K lippel-Feil syndrome (KFS) generally includes any form of congenital cervical vertebral fusion anomaly. ${ }^{1}$ The classic clinical triad, seen in $52 \%$ of KFS, includes a short neck, low hairline, and limitation of movement of the neck. ${ }^{2}$ Several congenital neurologic abnormalities and neurologic complications have been described in association with KFS including syringomyelia, agenesis of the corpus callosum, meningocele, and spinal stenosis.

\section{Case Reports}

\section{Case 1}

A 3-year-old white boy presented with a 3-week history of intermittent irritability, headaches, and dizziness associated with early morning vomiting. The child was diagnosed at the age of 11 months with KFS with fusion of the C2-C4 vertebral bodies (Fig 1). Genetic evaluation was otherwise normal, and no other associated anomalies in the central nervous system were found. CT and MR imaging were performed, which revealed a midline cystic posterior fossa mass with obstructive hydrocephalus and mild associated cerebellar tonsillar herniation.

At craniotomy, a cavity was found containing white fibrous, cheesy, purulent material with interspersed hairs. The cyst was excised and submitted for pathologic examination. Two-year follow-up MR imaging revealed postoperative changes without evidence of recurrent or residual tumor or hydrocephalus.

\section{Case 2}

A 12-month-old Hispanic girl presented with a 3-week history of fever, vomiting, and poor oral intake. The mother noted an enlarging occipital mass since the child was 5 months old, which had spontaneously drained a thick, white, paste-like material when the child was 8 months old. On transfer from an outside institution, CT and MR imaging were performed (Fig 2). Skeletal survey showed several hemivertebrae in her thoracic spine and fusion of several cervical vertebrae, particularly posteriorly.

Physical examination of the neck revealed a 4-cm, well-circumscribed, soft, slightly mobile, nontender mass in the occipital region.

Received April 20, 2007; accepted April 27.

From the University of Missouri-Kansas City School of Medicine (V.V.P., L.H.L., L.S.), Kansas City, Mo; Departments of Radiology (L.H.L.) and Pathology (L.S.), Children's Mercy Hospital and Clinics, Kansas City, Mo; and the Department of Neuroradiology (M.C., B.Y.H.), University of North Carolina-Chapel Hill, Chapel Hill, NC.

Please address correspondence to Vinay V. Pai, University of Missouri-Kansas City, One Shadewell Court, Danville, CA 94506; e-mail: vinaypai08@gmail.com

DOI 10.3174/ajnr.A0711
Suboccipital craniotomy with resection of the posterior fossa (cerebellar) mass demonstrated copious purulent fluid within both the cerebellar lesion and the subcutaneous abscess, which were connected by a sinus tract. On microscopic examination, the mass was diagnostic of a typical dermoid cyst. KFS was diagnosed based on the cervical spine radiographic findings and the presence of the dermoid. Postoperatively, she developed a posterior fossa pseudomeningocele, which gradually resolved, but otherwise did well.

\section{Case 3}

A 2-year-old African American boy with a history of KFS presented with epistaxis, frontal headaches, and a 6-month history of a small occipital, hairless, nontender lump with drainage of thick, creamy material the day before. The child was afebrile with normal vital signs. CT scan showed a soft tissue swelling over the occiput with an underlying skull defect connected to an intracranial cystic lesion, which was confirmed on MR imaging (Fig 3).

On occipital craniotomy, gross examination revealed a milky substance overlying a multiloculated mass of soft and firm areas, hair, and fat. Microscopic examination was typical for dermoid cyst with chronic inflammatory infiltrate. The postoperative course was uneventful, without residual tumor on postoperative MR imaging.

\section{Discussion}

In 1936, the relationship between posterior fossa dermoid tumors with cervical fusion anomalies such as the Klippel-Feil anomaly was first recognized. ${ }^{1}$ Since then, approximately 18 other cases of this rare association have been reported. ${ }^{3-6}$ Dermoid cysts are considered benign, and in all reported cases the cysts were well encapsulated on surgical removal. However, a case of squamous cell carcinoma dedifferentiation has been reported, and a few cases of rupture of the cyst leading to aseptic meningitis have been described. , $^{3,7}$

Several hypotheses have been proposed to explain the embryologic association of a posterior fossa dermoid and KFS. Failure of segmentation of the cervical sclerotomes leads to the Klippel-Feil anomaly and occurs after the formation of the entire neuraxis. The related failure of cleavage of ectoderm from neuroectoderm resulting in entrapment of dermal elements within the closing neural tube may contribute to the association of Klippel-Feil anomalies and dermoid cysts. ${ }^{2}$ Other proposed theories include overdistention of the neural tube resulting in distortion of the somites and reduced expressivity of the Hox or Pax genes, the highly conserved DNA sequences that control the development of the intervertebral disks. ${ }^{8}$ A mechanical basis to explain the relationship between 

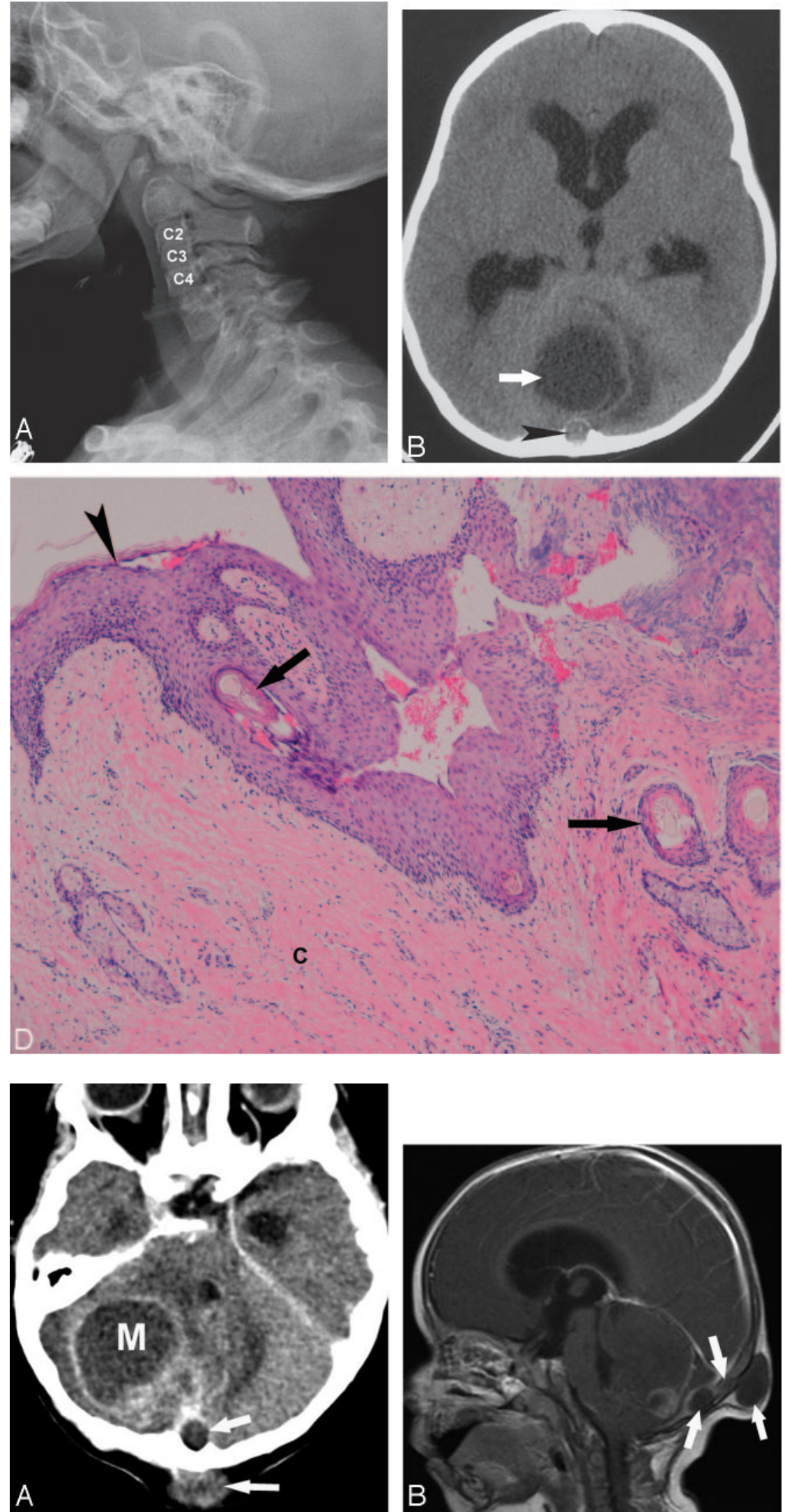

these abnormalities is that during the formation of the cephalic and cervical brain flexures, a shortening of the cervical spine because of a reduction or fusion in the number of somites may result in altered tissue tension, which could lead to entrapment of dermal elements. ${ }^{9}$

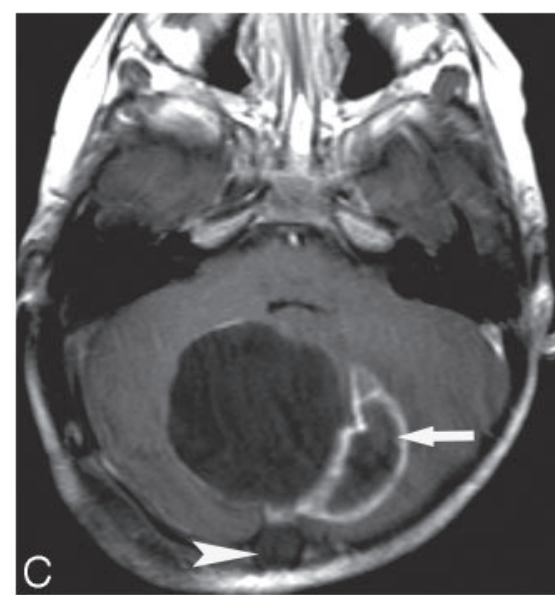

Fig 1. Posterior fossa dermoid and Klippel-Feil deformity in a 3-year-old boy. $A$, Lateral cervical spine radiograph shows a C2-C4 block vertebrae. B, Axial cranial CT without contrast demonstrates a midline posterior fossa mass (arrow), with mild surrounding low-attenuation halo of edema and a small focus of extension into the occipital bone (arrowhead). C, Axial T1-weighted MR image with contrast confirms the midline, cystic-appearing mass with peripheral enhancement (arrow) and extension through the midline occipital bone (arrowhead). D, Microscopic specimen of the benign dermoid cyst reveals keratinized squamous epithelial lining (arrowhead) with pilosebaceous glands and hair follicles with hair shafts (arrows). The cerebellar tissue (c) shows extensive inflammatory infiltrates (hematoxylin-eosin stain, original magnification X400).

Fig 2. Posterior fossa dermoid and Klippel-Feil deformity in a 12-month-old girl. A, Axial cranial CT with contrast shows a right heterogeneous posterior fossa mass with peripheral enhancement. Note that this mass $(M)$ extends beyond the midline and posterior through the occipital bone (arrows) into the occipital soft tissues. B, Sagittal T1-weighted image with gadolinium shows obstructive hydrocephalus. Note that the posterior fossa dermoid cyst extends through the occipital bone by a sinus tract (arrows).

Patients with KFS and posterior fossa dermoid cysts present with a variety of signs and symptoms, which may be attributed to both craniovertebral bony anomalies and increased intracranial pressure secondary to mass effect. It is interesting to note that, though seen in a minority of patients, 


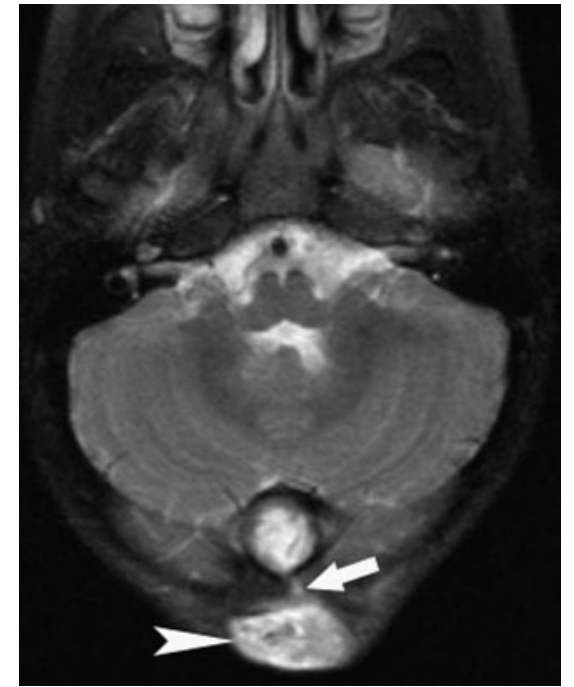

Fig 3. Posterior fossa dermoid and Klippel-Feil deformity in a 2-year-old boy. Axial T2-weighted image demonstrates a midline hyperintense intracranial mass with sinus tract (arrow) extending through the occipital bone forming an occipital scalp soft tissue mass (arrowhead).

the presence of mirror movements is more likely to be seen in children with Klippel-Feil anomalies and neuroschisis. ${ }^{1}$ The exact anatomic basis of mirror movements remains in question, but this sign may be a clue to an occult posterior fossa dermoid in children with Klippel-Feil deformity. ${ }^{1}$

To our knowledge, there have been only 7 reported cases that describe KFS in association with a posterior fossa dermoid cyst that extends through a skull defect into the occipital bone and presents as a suboccipital subcutaneous mass. ${ }^{3-5}$ Of the 3 patients presented herein, 2 presented in this manner. The persistence of a sinus tract allows access to the intracranial space, increasing susceptibility to meningitis. Patients with KFS and posterior fossa dermoids should be carefully scrutinized with imaging because both the mass and the sinus tract must be surgically removed.

Neuroimaging with CT and MR, in addition to a clinical history of Klippel-Feil anomaly, helps to make the diagnosis of posterior fossa dermoid cysts. On CT, dermoid cysts lack cen- tral enhancement and, when uncomplicated by infection, are typically hypoattenuated with attenuation ranging from 0 to $-150 \mathrm{HU}^{6}$ On MR imaging, signal intensity is variable depending on predominant contents within the mass and the presence or absence of superimposed infection. Both increased and decreased signal intensities have been reported on TI- and T2-weighted MR imaging sequences. ${ }^{6}$ Diffusionweighted MR imaging is useful to differentiate epidermoid tumors from arachnoid cysts and CSF collections, which are otherwise indistinguishable. ${ }^{7}$ Specifically, epidermoid tumors show marked increased signal intensity on diffusion-weighted images, whereas arachnoid cysts and CSF collections are generally hypointense following signal intensity of CSF. ${ }^{10}$

Dermoid cysts should be suspected when recurrent meningitis, mirror movements, or a midline posterior fossa mass at the craniocervical junction, especially with an associated occipital soft tissue mass, with or without drainage are found in patients with KFS. ${ }^{6}$

\section{References}

1. Royal SA, Tubbs RS, D’Antonio MG, et al. Investigations into the association between cervicomedullary neuroschisis and mirror movements in patients with Klippel-Feil syndrome. AJNR Am J Neuroradiol 2002;23:724-29

2. Muzumdar D, Goel A. Posterior cranial fossa dermoid in association with craniovertebral and cervical spinal anomaly: report of two cases. Pediatr $\mathrm{Neu}$ rosurg 2001;35:158-61

3. Oertel J, Piek J, Müller JU, et al. Posterior fossa squamous cell carcinoma due to dedifferentiation of a dermoid cyst in Klippel-Feil syndrome case illustration. J Neurosurg 2002;97:1244

4. Chandra PS, Gupta A, Mishra NK, et al. Association of craniovertebral and upper cervical anomalies with dermoid and epidermoid cysts: report of four cases. Neurosurgery 2005;56:E1155; discussion E1155

5. Hinojosa M, Tatagiba M, Harada K, et al. Dermoid cyst in the posterior fossa accompanied by Klippel-Feil syndrome. Childs Nerv Syst 2001;17:97-100

6. Sharma MS, Sharma BS, Yadav A, et al. Posterior fossa dermoid in association with Klippel-Feil syndrome-a short report. Neurol India 2001;49:210-12

7. Aksoy FG, Aksoy OG, Gomori JM. Klippel-Feil syndrome in association with posterior fossa suboccipital dermoid cyst. Eur Radiol 2001;11:142-44

8. Gardner WJ. Klippel-Feil syndrome, iniencephalus, anencephalus, hindbrain hernia and mirror movements: overdistention of the neural tube. Childs Brain 1979;5:361-79

9. Kennedy PT, McAuley DJ. Association of posterior fossa dermoid cyst and Klippel-Feil syndrome. AJNR Am J Neuroradiol 1998;19:195

10. Nguyen JB, Ahktar N, Delgado PN, et al. Magnetic resonance imaging and proton magnetic resonance spectroscopy of intracranial epidermoid tumors. Crit Rev Comput Tomogr 2004;45:389-427 